Classification

Physics Abstracts

$68.10 \mathrm{C}-82.65 \mathrm{D}-68.55-61.14 \mathrm{H}$

\title{
Incomplete wetting of methane on graphite at low temperatures
}

\author{
J. Krim (*), J. M. Gay, J. Suzanne and E. Lerner $\left({ }^{* *}\right)$ \\ Laboratoire Surfaces-Interfaces, U.A. CNRS 794, Département de Physique, Faculté des Sciences de Luminy, \\ Case 901, 13288 Marseille Cedex 9, France
}

(Reçu le 7 avril 1986, accepté le 3 juin 1986)

\begin{abstract}
Résumé. - Le mouillage du graphite par le méthane a été étudié par diffraction d'électrons lents et d'électrons rapides dans le domaine de température $14-40 \mathrm{~K}$. Un mouillage incomplet (formation de cristallites) est apparu sur toute cette gamme de température pour des films d'épaisseur supérieure à 15 couches. Les chaleurs isostériques d'adsorption des $2^{e}, 3^{e}$ et $4^{e}$ couches mesurées sont en accord avec celles déterminées par ailleurs à $77 \mathrm{~K}$.
\end{abstract}

\begin{abstract}
The wetting behaviour of methane on graphite has been studied by means of low energy electron diffraction and reflection high energy electron diffraction in the temperature range 14-40 K. Incomplete wetting, or crystallite formation, is detected over the entire temperature range for films with thicknesses of 15 or more layers. Isosteric heats of adsorption are reported for the $2 \mathrm{nd}$, 3rd and 4th adsorbed layers, and agree with earlier measurements recorded at $77 \mathrm{~K}$.
\end{abstract}

\section{Introduction.}

The growth mode of a film on a solid substrate is an important issue in surface science concerning a wide range of fundamental and applied problems. Macroscopically, a film may form a uniform layer on a substrate or it may form crystallites (or droplets). The former behaviour is termed complete wetting, type 1 or Frank van der Merwe growth and the latter incomplete wetting, type 2 or Stranski-Krastanov growth [1-3]. It is generally observed that the crystallites associated with the latter growth mode coexist with a layer of the same material whose thickness is finite and of microscopic dimensions. Wetting behaviour can be temperature dependent and a system may exhibit a wetting transition from one growth mode to the other at the wetting temperature $T_{\mathrm{w}}$ [4].

Theoretical interest has increased dramatically within the last several years with the introduction of microscopic models of film growth $[2,5]$. The earliest of these models considered spherical particles adsorbed on attractive, planar substrates and characterized the wetting behaviour as a function of the relative strength of the adsorbate-adsorbate and

(*) Present address : Physics Department, Northeastern University, Boston, MA 02115, USA.

(**) Present address: Instituto de Fisica, Universidade Federal do Rio de Janeiro, Cidade Universitaria, 21944RJ, Brazil. adsorbate-substrate interactions. They confirmed traditional belief that at $T=0 \mathrm{~K}$, films on strongly attractive substrates exhibit complete wetting, while on less attractive substrates incomplete wetting will occur. Subsequent experiments showed, however, that type 1 growth of solid films at low temperature is limited to a narrow range of relative substrate attraction, type 2 growth being observed for both stronger and weaker relative substrate attraction [6]. This « reentrant » nature of type 2 growth on a scale of relative substrate attraction is illustrated in table $\mathrm{I}$, which compares atom-atom and atom-substrate interactions for the various gases which have been studied to date on graphite (including this work). The gauge of relative interaction strength listed is the ratio $u_{1} / h_{0}$ which was proposed by Bienfait $e t$ al. [7] : $u_{1}$ is the low coverage atom binding energy, and $h_{0}$ is the $0 \mathrm{~K}$ cohesive energy of the bulk adsorbate.

It was quickly shown that a strongly attractive substrate could compress the first few layers to produce significant structural mismatch with closepacked planes of bulk solid inducing a strain in the film that might prevent it from completely wetting the substrate [8]. Here, the effects of substrate modulation and non-spherical adsorbate molecules were not invoked to produce a mismatch of film with the bulk, although they are certainly present in some systems. Previous investigations of the system $\mathrm{CH}_{4}$ /graphite give evidence for complete wetting in the temperature range $T>64 \mathrm{~K}$, as at least six 
Table I. - Summary of previous and present experimental results for low temperature wetting behavior on a scale of relative substrate attraction (Ref. [7]).

\begin{tabular}{l|cccccccccc}
\multicolumn{1}{c|}{ Adsorbate } & $\mathrm{C}_{2} \mathrm{H}_{4}$ & $\mathrm{CF}_{4}$ & $\mathrm{O}_{2}$ & $\mathrm{Xe}$ & $\mathrm{Kr}$ & $\mathrm{Ar}$ & $\mathrm{N}_{2}$ & $\mathrm{CH}_{4}$ & $\mathrm{Ne}$ & $\mathrm{He}$ \\
\hline$u_{0} / h_{0}$ & $0.4-0.7$ & 0.7 & 1.02 & 1.18 & 1.15 & 1.28 & 1.30 & 1.43 & 1.55 & 20 \\
Mode & 2 & 2 & 2 & 1 & 1 & 1 & 2 & 2 & 2 & 2 \\
\hline
\end{tabular}

atomic layers are observed to condense on the surface with no indication of cluster formation [9]. This behaviour is reported to give way to a narrow regime of incomplete wetting as temperature is increased to $0.2 \mathrm{~K}$ below the triple point, a direct consequence of the fact that methane solid and liquid surface tensions are unequal [10]. While there have been no previous studies of wetting at low temperature, the lattice parameters of bulk methane [11] and monolayer films on graphite [12] have been measured, and reveal, before second layer formation, a compression of the monolayer with respect to the (111) bulk plane of less than $1.5 \%$ within our experimental temperature range. This mismatch being relatively small, one might expect $\mathrm{CH}_{4}$ to exhibit complete wetting at low temperature, despite its location well within the reentrant type 2 regime of the relative substrate interaction scale. On the other hand, one might expect the interaction scale to hold true, and look for a transition from type 1 to type 2 behaviour in the temperature range $0<T<64 \mathrm{~K}$, or for the formation of clusters at coverages higher than six atomic layers.

We were thus motivated to study this system and report here our results, obtained via low energy electron diffraction (LEED) and reflection high energy electron diffraction (RHEED) : incomplete wetting was observed over the entire temperature range studied $(14 \mathrm{~K}<T<40 \mathrm{~K})$. However, crystallite formation was detected only in films with statistical thicknesses of 15 layers or more. Methane on graphite approaches complete wetting more than any of the other type 2 systems studied to date at low temperature, a result entirely consistent with the small lattice mismatch parameter which characterizes this system.

\section{Experimental apparatus and technique.}

RHEED is a technique well adapted to the observation of the various modes of growth of surface films. For a flat, layered film the diffraction pattern consists of parallel, slightly modulated streaks normal to the substrate plane, whereas small bulk crystallites produce sharp spots. If a few molecular layers coexist with crystallites, the diffraction pattern exhibits streaks together with sharp spots [13].

Our apparatus includes, in addition to the RHEED system, a low energy electron diffractometer (LEED) which allows us to measure the pressures of condensation of the first layers and to estimate the thickness of the adsorbed film. We plot photometric isotherms by recording the 10 graphite spot attenuation of the LEED pattern intensity as methane condenses on the surface. This condensation occurs under quasi-equilibrium conditions by slowly increasing the pressure in the chamber. However, for temperatures where equilibrium condensation occurs at lower than $1 \times 10^{-8}$ torr, $\mathrm{CH}_{4}$ is deposited kinetically on the surface under a constant gas pressure of $1 \times 10^{-8}$ torr. Here, the isotherm is recorded by plotting graphite spot attenuation versus deposition time [14].

Graphite surface quality was monitored by observing the LEED pattern of the bare graphite, methane and/or $S_{1}$ ethane monolayers [15] before and after the experimental runs to check for possible changes in surface adsorption characteristics. Purity of the methane gas (Messer-Griesheim 99.995\% grade) was monitored by a mass spectrometer. The substrate was a $3 \times 3 \mathrm{~mm}^{2}$ cleaved crystal of natural graphite mounted on a stage which could be cooled to $14 \mathrm{~K}$ and regulated to better than $0.1 \mathrm{~K}$. Pressure was measured with a Bayard-Alpert gauge, calibrated by extrapolation of measurements of $\mathrm{CH}_{4}$ pressure in the $10^{-3}-10^{-1}$ torr range with a Baratron gauge. This system has been described in detail elsewhere [16]. For these studies we have modified the sample holder so as to allow variation of the RHEED azimuthal incidence angle by $\pm 30^{\circ}$. We have not applied a thermo-molecular correction to the measured pressure, as explained in references 15 and 18.

\section{LEED results.}

Over the entire temperature range studied, the first $\mathrm{CH}_{4}$ layer condenses at pressures well below $5 \times 10^{-9}$ torr, preventing us from studying it under equilibrium conditions. This was also the case for the second and third layers for approximately $T<30 \mathrm{~K}$.

For $35 \mathrm{~K}<T<40 \mathrm{~K}$, photometric isotherms of the 10 graphite spot were recorded as a function of $\mathrm{CH}_{4}$ vapour pressure under equilibrium conditions. A typical LEED adsorption isotherm recorded at $36.2 \mathrm{~K}$ is shown in figure 1 . A sudden drop of the intensity is observed as soon as the gas is admitted. It corresponds to the adsorption of the first layer, whose equilibrium condensation pressure at this temperature is $\ll 10^{-11}$ torr [18]. Three other steps occur at $6.3 \times 10^{-8}, 2.6 \times 10^{-7}$ and $4.2 \times 10^{-7}$ torr respectively. The first two correspond to the condensation of the second and third layers. We are unable to say whether the last one is due to the condensation of a fourth layer or to formation of bulk methane. In any case, these transitions are expected to be very 


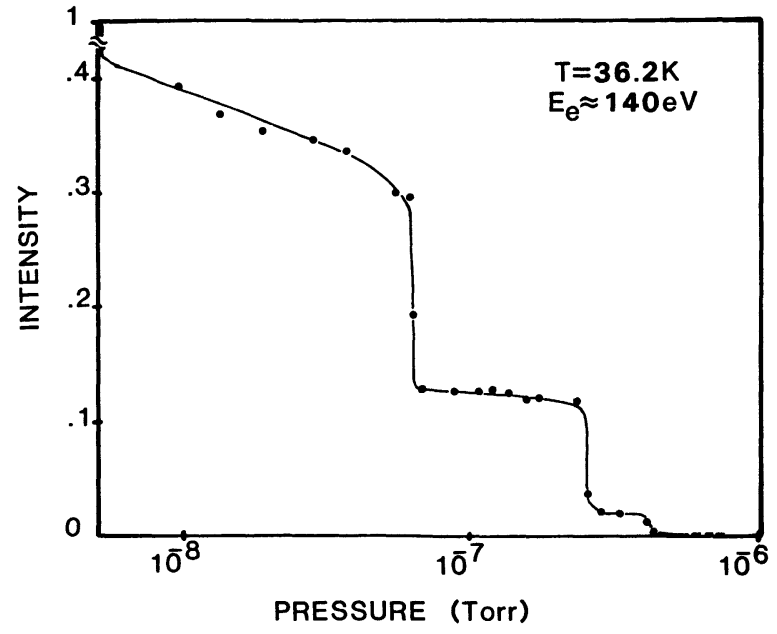

Fig. 1. - Photometric LEED isotherm of methane on graphite at $36.2 \mathrm{~K}$ recorded continuously over the pressure range under quasi-equilibrium conditions. The points represent true equilibrium conditions. The electron beam energy is $140 \mathrm{eV}$. An intensity value of 1 corresponds to that of the 10 graphite spot before any adsorption, while 0 corresponds to the background intensity. The steps at $6.3 \times 10^{-8}, 2.6 \times 10^{-7}$ and $4.2 \times 10^{-7}$ torr correspond to the condensation of the second, third and fourth layers. The equilibrium condensation pressure of the first layer is well below $1 \times 10^{-10}$ torr.

close. We have plotted the pressures of the three steps observed on six photometric isotherms on a log $P$ versus $1 / T$ phase diagram (Fig. 2 , lines 2,3 and 4 ) along with other independently measured $\log P$ versus $1 / T$ lines $[18,19]$. The heats of condensation of the second, third and fourth layers can be deduced from the slope of these Clausius-Clapeyron lines [20]. These are listed in table II along with those reported by Thomy and Duval [19], recorded at higher temperatures. In light of the large difference in temperature and pressure ranges studied, the two sets of results are quite close. In addition, all of our isotherms recorded under equilibrium conditions obey the Frankel-Halsey-Hill relation

$$
\operatorname{Ln} P_{n} / P_{0}=-\alpha / T n^{3}
$$

for $\mathrm{n}=2$ and 3 , where $P_{n}$ and $P_{0}$ are respectively the condensation pressure of the $n^{\text {th }}$ layer and bulk. It is impossible to infer any deviation from the $n^{-3}$ law due to film and substrate interaction, given the limited number of experimental points. The value $\alpha$ obtained $\left(615 \pm 44 \mathrm{~K}\right.$ layer $\left.^{3}\right)$ is consistent with that reported by Thomy et al. [19] for data recorded at $77 \mathrm{~K}\left(590 \mathrm{~K}\right.$ layer $\left.^{3}\right)$. These values are somewhat larger than the theoretical estimate obtained using Vidali and Cole approximation [21] extended by Rauber et al. [22], that is $492 \mathrm{Klayer}^{3}$. In this calculation, we have assumed a constant interlayer spacing equal to $3.4 \AA$, that is the distance between (111) planes of bulk $\mathrm{CH}_{4}$ around $T=30 \mathrm{~K}$ [11].

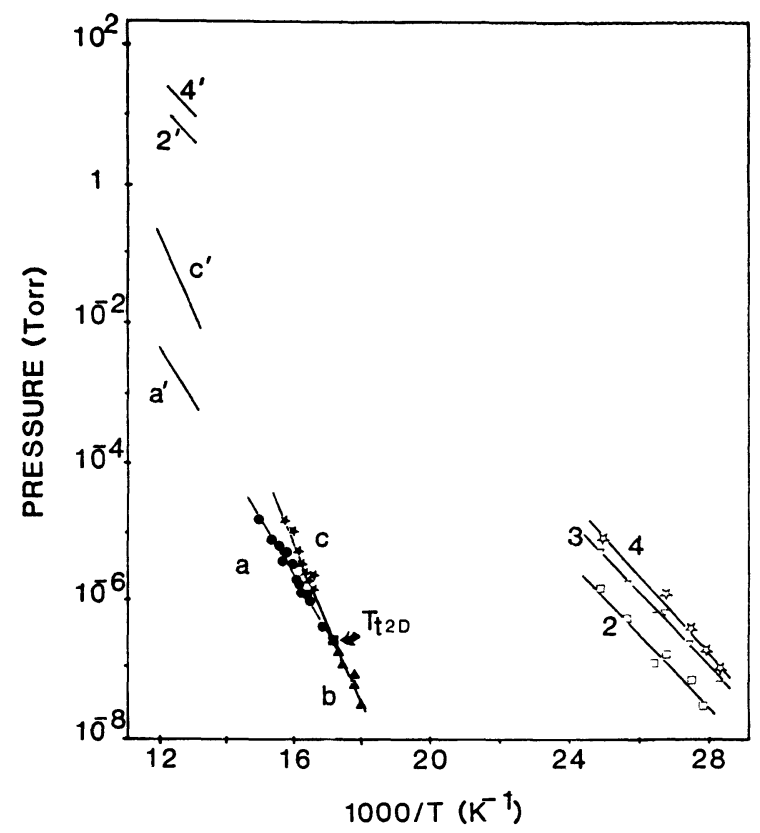

Fig. 2. - Log $P$ versus $1 / T$ phase diagram for the second, third and fourth layers (lines 2,3 and 4 , respectively), along with the Clapeyron lines $2^{\prime}$ and $4^{\prime}$ for the second layer and bulk condensation [19]. Lines a, $a^{\prime}, b, c$ and $c^{\prime}$ concern transitions in the monolayer regime [18].

Table II. - Isosteric heats of adsorption for the $2 n d$, $3 r d$ and 4 th layers of methane on graphite $(\mathrm{kcal} / \mathrm{mol})$.

\begin{tabular}{c|c|c} 
& This work & Ref. [19] \\
\hline Layer & $35 \mathrm{~K}<T<40 \mathrm{~K}$ & $77 \mathrm{~K}<T<84 \mathrm{~K}$ \\
\hline 2 & $2.4 \pm 0.2$ & 2.38 \\
3 & $2.5 \pm 0.2$ & undetermined \\
4 & $2.5 \pm 0.3$ & $2.33\left(^{*}\right)$ \\
\hline
\end{tabular}

$\left(^{*}\right)$ For coverages $\gg 5$ layers.

At temperatures below $30 \mathrm{~K}$ where the bulk vapour pressure is less than $5 \times 10^{-9}$ torr we are unable to record data under equilibrium conditions. Isotherms are recorded as a function of deposition time. Five such kinetic isotherms are shown in figure 3. For $T \geqslant 16 \mathrm{~K}$ we observe three discontinuities corresponding to the condensation of three layers of methane. Again, for fourth and higher layers the signal is lost to the background. The intensity of the graphite spot after condensation of each layer $(41 \%, 14 \%$ and $4 \%$ of the initial intensity) agrees with that observed for the respective layers under equilibrium conditions. The kinetic isotherms allow us to estimate the condensation rate to be approximately 0.17 layers $/ \mathrm{min}$ at $1 \times 10^{-8}$ torr, which gives a sticking coefficient of 0.37 . It is this rate which we employ to determine statistical film thicknesses for coverages greater than three layers assuming a constant sticking coefficient. At $\simeq 14 \mathrm{~K}$ there is an abrupt change in the form of the kinetic isotherms : the second and third layers disappear in 


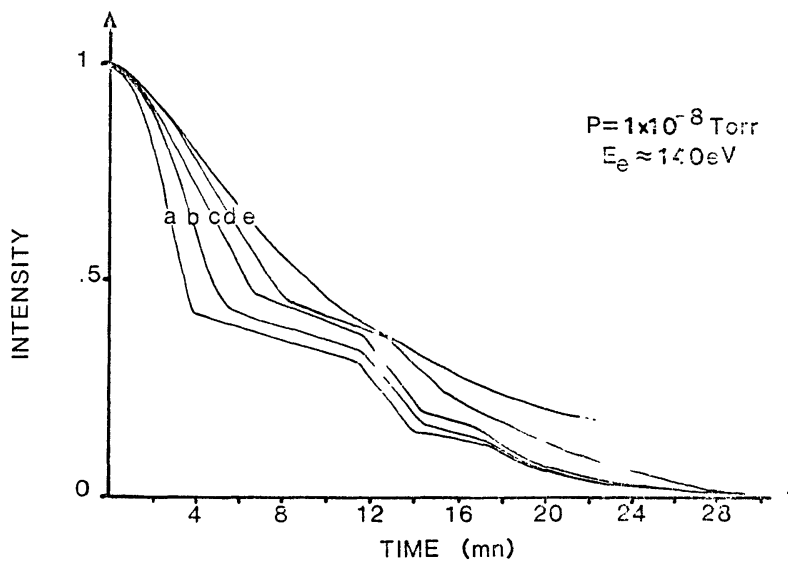

Fig. 3. - Photometric kinetic LEED isotherms at (a) $30 \mathrm{~K}$, (b) $21 \mathrm{~K}$, (c) $19 \mathrm{~K}$, (d) $16 \mathrm{~K}$ and (e) $14 \mathrm{~K}$ recorded under a constant methane flux of $1 \times 10^{-8}$ torr. The intensities 1 and 0 correspond to that of bare graphite, and the background, respectively.

succession and the total attenuation as a function of time greatly decreases. No changes are observed in the first layer characteristics.

This behaviour is somewhat similar to that observed in the system $\mathrm{CF}_{4}$ on graphite [14] and one is tempted to interpret this as a transition from complete to incomplete wetting or, at least, a thin film-thick film transition. On the other hand, it may be due to a change in the kinetics of reorganization of the adsorbed film. Such a change seems unlikely however within this narrow temperature range (14$16 \mathrm{~K})$. Given our temperature uncertainty for experiments performed below $20 \mathrm{~K}( \pm 3 \mathrm{~K})$, it may be related to the transition observed by Kim et al. [23] near $11 \mathrm{~K}$.

\section{RHEED results.}

The RHEED experiments were performed at an azimuthal incidence angle that corresponds to the 120 direction of the beam with respect to the graphite reciprocal lattice and is favorable for observation of films adsorbed in the $\sqrt{3} \times \sqrt{3} \mathrm{R} 30^{\circ}$ position. These experiments went as follows : the sample was adjusted so that a photometric LEED isotherm could be recorded simultaneously with the RHEED observations. For temperatures between 14 and $30 \mathrm{~K}$, the pressure was set at $1 \times 10^{-8}$ torr, and we proceeded to record photometric isotherms such as those described in section 2 , with the RHEED electronics turned off. Employing the LEED information to estimate film thickness, we turned on the RHEED electronics approximately once every four statistical layers for a period of 510 seconds to view or photograph the RHEED pattern. We then turned off the electronics and waited for more layers to form. We could not observe a continuous RHEED pattern : the incoming beam apparently desorbed the methane mole- cules. We found no evidence however for substrate contamination due to beam-induced cracking of the methane molecules, if indeed this was occurring [24]. At higher temperatures $(30-40 \mathrm{~K})$, data were recorded in a similar manner with the exception that higher pressures were required to condense the film layers. We observed essentially the same behaviour over the entire temperature range studied.

For the initial film layers, we observed streaks which were easily indexed to the 2D hexagonal $\mathrm{CH}_{4}$ reciprocal lattice as $10,20, \overline{10}$ and 20 . First faint and uniform (1-2 layers), they became more intense and increasingly modulated as coverage increased (3-15 statistical layers). This modulation results from the finite penetration of the grazing-incidence beam. A typical photograph of a ten statistical layer streak pattern is shown in figure 4A. From these results we conclude that the film is at least 15 statistical layers thick for temperatures above $14 \mathrm{~K}$. To reconcile this result with that of the kinetic LEED isotherm at $14 \mathrm{~K}$, we may reasonably assume a slight heating of the surface by the RHEED beam.

At higher coverages ( $>15$ statistical layers), the behaviour was quite different. At these coverages sharp spots appeared on the streaks indicating that bulk crystals were present (Fig. 4B). Due to the difficulties associated with the RHEED electron beam desorption of the film, we were unable to determine quantitatively the film thickness at which spots first appeared, and in particular its dependence
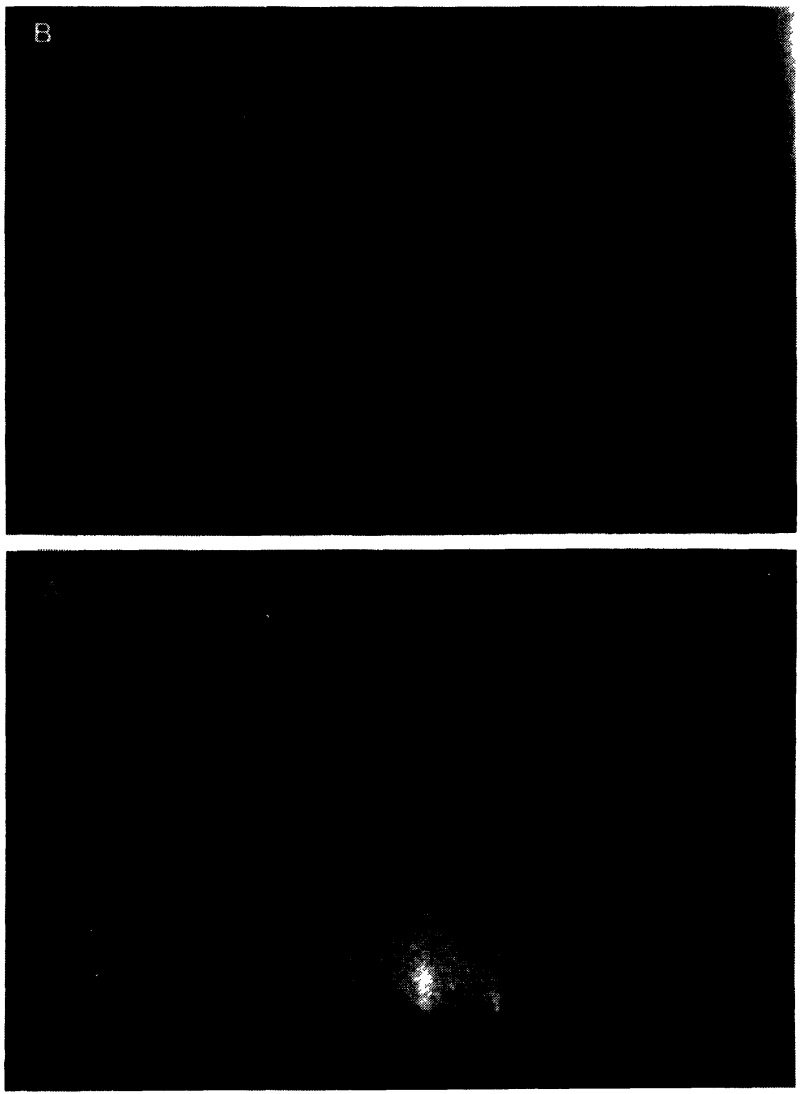

Fig. 4. - RHEED patterns for (A) 10 statistical layers and (B) 15 statistical layers. 
on temperature. Films which were twenty statistical layers thick always showed crystallite formation over the entire temperature range studied. We observed no « reorganization effects » as reported by Gay et al. [14] for $\mathrm{CF}_{4}$ films on graphite : a RHEED pattern due to the methane overlayer was clearly present for all film thicknesses studied. We did observe on occasion that spots would appear in a RHEED pattern upon cooling the film, indicating that the film thickness when the crystallites formed was indeed temperature dependent, and increased with increasing temperature. We take this latter result to be a strong indication that the adsorbed films were in equilibrium. Again, no precise information on film thickness was available on account of the electron beam desorption of the film.

We observed no wetting transitions. At $64 \mathrm{~K}$, over six layers were observed [9], a result consistent with our observations. Our results do, however, raise the question of whether this may be interpreted as complete wetting and show that extreme caution must be employed in assigning the term " complete wetting " to any system on the basis of a relatively large number of layers alone.

\section{Discussion.}

To date, only $\mathrm{Xe}, \mathrm{Ar}$ and $\mathrm{Kr}$ have been claimed to exhibit type 1 growth on graphite at low temperature [6]. However, this result was deduced from RHEED experiments on films which were only ten statistical layers thick. In order to verify that crystallites did not form at some higher coverage, we repeated the measurements for $\mathrm{Xe}$ at $49 \mathrm{~K}$ and $5 \times 10^{-9}$ torr. We confirmed the results of Seguin et al. that the ten layer pattern shows modulated streaks, and then continued to condense further layers to check for crystallite formation. Increasing the pressure in the chamber first to $5 \times 10^{-8}$ and then $1 \times 10^{-7}$ torr, we condensed approximately two hundred fifty statistical layers of xenon, and observed no change in the RHEED pattern : modulated streaks were still clear and no spots had appeared. This behaviour is precisely what is to be expected from a type 1 system, and in any case is markedly different from that observed for methane under the identical experimental conditions. In addition, the results are in agreement with theoretical predictions for the wetting behaviour of solid films in the reentrant type 2 regime on a scale of $u / v$ : systems such as methane on graphite which fall close to the type 1 regime should form relatively thick films before bulk crystallites condense at low temperature. Our results certainly do not rule out a thick-thin film transition below $16 \mathrm{~K}$, evidence for which has been observed in recent heat capacity measurements of this same system [23]. We cannot illuminate here the precise temperature relationship of the film thickness at coexistence nor can we state definitively the macroscopic form of methane on graphite. Given the RHEED experimental resolution $(\simeq 2 \%)$, we cannot estimate if there is any lattice mismatch between the uniform film and the dense (111) plane of bulk. As further studies, we would suggest experiments which would bridge the gap between our microscopic results and the macroscopic world. Specifically, we suggest optical and/or contact angle studies of solid and liquid methane films on graphite.

\section{Acknowledgments.}

This work has been supported by a NATO postdoctoral fellowship awarded to J. K. in 1984. Two of us, J. K. and E. L. kindly acknowledge Professor M. Bienfait for the hospitality of the Laboratoire Surfaces-Interfaces.

\section{References}

[1] Dash, J. G., Phys. Rev. B 15 (1977) 3136.

[2] PANDIT, R., SCHICK, M. and Wortis, M., Phys. Rev. B 26 (1982) 5112.

[3] BAUER, E. and POPPA, H., Thin Solid Films 12 (1972) 167

[4] Cahn, J. W., J. Chem. Phys. 66 (1977) 3667.

[5] Sullivan, D. E., Phys. Rev. B 20 (1979) 3991.

[6] Seguin, J. L., Suzanne, J., Bienfait, M., Dash, J. G. and Venables, J. A., Phys. Rev. Lett. 51 (1983) 122

[7] Bienfait, M., Seguin, J. L., Suzanne, J., Lerner, E., Krim, J. and DASH, J. G., Phys. Rev. B 29 (1984) 983.

[8] Ebner, C., RotTman, C. and Wortis, M., Phys. Rev. B 28 (1983) 4186.

Muirhead, R. J., DASh, J. G. and Krim, J., Phys. Rev. B 29 (1984) 5074.

Huse, D. A., Phys. Rev. B 29 (1984) 6985 ;

GiTTES, F. T. and SCHICK, M., Phys. Rev. B 30 (1984) 209

[9] Hamilton, J. J. and Goodstein, D. L., Phys. Rev. B 28 (1983) 3838.
[10] Pettersen, M. S., Lysek, M. L. and Goodstein, D. L. (preprint).

[11] Greer, S. C. and Meyer, L., Z. Angew. Phys. 27 (1969) 198 ;

ManzheliI, V. G., Tolkachev, A. M. and Gavrilko, V. G., J. Phys. Chem. Solids 30 (1969) 2759.

[12] Vora, P., Sinha, S. K. and Crawford, R. K., Phys. Rev. Lett. 43 (1979) 704.

[13] BAUER, E., in Techniques of Metal Research, edited by R. F. Bunshah (Interscience, New York), 1969, Vol. 2, Pt. 2, p. 501.

[14] Suzanne, J., Seguin, J. L., Bienfait, M. and Lerner, E., Phys. Rev. Lett. 52 (1984) 637 ;

GAY, J. M., Bienfait, M. and SuzAnne, J., J. Physique 45 (1984) 1497.

[15] Suzanne, J., GAY, J. M. and WANG, R., Faraday Symp. Chem. Soc. 20 (1985) to be published.

[16] Venables, J. A., Seguin, J. L., SuZanNe, J. and Bienfait, M., Surf. Sci. 145 (1984) 345. 
[17] Fain, S. C., in Chemistry and Physics of Solid Surfaces $I V$, edited by $\mathrm{R}$. Vanselow and $\mathrm{R}$. Howe (Springer-Verlag, New York) 1982, p. 207.

[18] Gay, J. M., Dutheil, A., Krim, J. and Suzanne, J., Surf. Sci. to be published.

[19] Тномy, A. and Duval, X., J. Chim. Physique 66 (1969) 1966 ; 67 (1970) 1101.

[20] LANDAU, L. D. and Lifshitz, E. M., Statistical Physics, 3rd edition, part 1, p. 256 ;
Larher, Y., J. Coll. Int. Sci. 37 (1971) 836.

[21] Vidali, G. and Cole, M. W., Surface Sci. 110 (1981) 10.

[22] Rauber, S., Klein, J. R., Cole, M. W. and BRUCH, L. W., Surface Sci. 123 (1982) 173.

[23] KiM, H. K., ZhaNG, Q. M. and ChaN, M. H. W. Faraday Symp. Chem. Soc. 20 (1985) to be published.

[24] Suzanne, J. and Bienfait, M., J. Physique Colloq. 38 (1977) C4-93. 